\title{
Reconstruction of superthermal electron velocity distribution function from electron cyclotron spectra at down-shifted frequencies in tokamak T-10
}

\author{
P.V. Minashin, A.B. Kukushkin, V.I. Poznyak \\ Tokamak Physics Institute, NRC “Kurchatov Insitute”, 123182 Moscow, Russia
}

\begin{abstract}
A model is developed to quantitatively interpret the electron cyclotron (EC) spectra in tokamak T-10 at frequencies lower than the first EC harmonic at the plasma edge. The model is based on the almost free propagation and isotropy of $\mathrm{EC}$ radiation in between the vacuum chamber wall and the cut-off zone in plasma. The inverse problem for the reconstruction of the velocity distribution function of superthermal electrons (in parallel and perpendicular momenta, and magnetic flux surfaces at the plasma edge) is formulated and solved. It is shown that in the Ohmic discharges in the tokamak T-10 the superthermal electrons are the trapped particles at the low magnetic field side of toroid. Their mean kinetic energy lies in the range $\sim 150-200 \mathrm{keV}$, and the density fraction is about $\sim 10^{-4}$ relative to the main plasma.
\end{abstract}

\section{Introduction}

Observations of electron cyclotron (EC) radiation spectra in tokamak T-10 simultaneously in the range of the first and second $\mathrm{EC}$ harmonics of thermal plasma $\mathrm{EC}$ emission, including relativistically downshifted frequencies for both harmonics, suggested the presence of a fraction of superthermal electrons with energies in the range up to few hundreds of $\mathrm{keV}$ [1]. The observed spectra did not depend on the angles of detected waves and on their polarization.

Observations of electron cyclotron radiation (ECR) spectrum in the low frequency range and the interpretation of its features with a contribution of superthermal electrons have been widely discussed in the literature, see, e.g., experiments in the Ohmic discharges (tokamak ASDEX-U [2]), ECRH and LHCD discharges (tokamaks ALCATOR [3], WT-3 [4], FT-2 [5]), though there the measurements were carried out at slightly higher frequencies. The characteristic feature of the experiments in tokamak T-10 is the smallness of the plasma density at the periphery (noticeably lower than such in large tokamaks), that allows the propagation of the $1^{\text {st }}$ harmonic O-mode EC radiation at the periphery (with increasing density the allowable region for this radiation is expelled from plasma).

Here we developed a model for quantitative interpretation of the above-mentioned measurements in tokamak T-10. The model is based on the almost free propagation and isotropic intensity of the $\mathrm{EC}$ radiation in between the vacuum chamber wall and the cut-off zone in plasma. This allows us to apply the calculation method formerly developed for the ECR power losses in tokamak-reactors where the radiation at high frequencies, responsible for the EC power losses, is trapped in between the vacuum chamber wall and the inner region of high optical opacity. We have formulated an 
inverse problem for the reconstruction of the superthermal electron velocity distribution function (VDF) in parallel and perpendicular momenta, and in magnetic flux surfaces at the plasma edge. The exact solution is obtained for the case of superthermal electrons localized within a thin layer at the plasma edge. The case is suggested by the measurements in tokamak T-10 [1], which revealed the dependence of the low-frequency edge of the radiation spectrum on the density at the periphery in agreement with the wave cut-off for the O-mode.

The problem of the reconstruction of the superthermal electron VDF from the observed ECR spectrum was treated in a number of papers, see, e.g., [3], [4], [6], [7]. The key element of these papers is the assumption of a certain functional type of the VDF with several fitting parameters. In this paper we solve an inverse problem without any assumptions on the functional type of the VDF. Such a freedom in solving the inverse problem is possible due to a strong dependence of the ECR spectrum for superthermal electrons in a nonuniform magnetic field in a tokamak on the radiation frequency in the range of relativistically down-shifted frequencies.

The calculations, carried out for the case of the Ohmic discharges in tokamak T-10, enable us to determine the type of the trajectory of the radiating electrons (either they are the trapped or circulating particles), mean kinetic energy and the ratio of superthermal electron density to the main plasma density at the plasma edge.

\section{Modeling of EC radiation spectrum}

We propose the following model to describe the observed ECR spectrum.

- Superthermal electrons are localized within $3 \mathrm{~cm}$ layer near the plasma edge (it is suggested by the experiment [1]).

- Almost vacuum limit for calculating the EC emission rate is used: Schott-Trubnikov formula, no refraction, the allowance for wave cut-off.

- Negligible absorption of ECR by the bulk plasma in the range of down-shifted frequencies.which do not fall in the range of thermal radiation by the cold plasma.

- ECR intensity is homogeneous and isotropic in angles in-between the vacuum chamber metal wall and the cut-off zone, due to multiple reflections of the radiation from the wall (this is suggested by the measurements: independence of spectrum from the polarization and from the location of radiation detector).

This allows us to apply the formalism formerly developed and implemented in the numeric codes for the ECR power losses in tokamak-reactors, CYTRAN [8] and CYNEQ [9-12], because in tokamak-reactors the radiation at high frequencies responsible for the EC power losses is trapped in between the vacuum chamber wall and the inner region of high optical opacity.

The radiation spectral temperature, $\mathrm{T}_{\mathrm{rad}}$, is defined in the standard way in terms of the observed intensity of the outgoing EC radiation, I:

$$
\mathrm{T}_{\mathrm{rad}}(\omega)=\frac{8 \pi^{3} \mathrm{c}^{2}}{\omega^{2}} \mathrm{I}(\omega)
$$

In the proposed model the intensity of the outgoing radiation is described by the following formula (here we assume no mixing of the modes, caused by wave reflection; the allowance for reflections give formulas with similar structure):

$$
\begin{gathered}
I(\omega)=I_{X}(\omega)+I_{O}(\omega), \\
I_{X, O}(\omega)=\frac{\int d \Omega_{\vec{n}} \int_{V_{x, O}^{\text {sec }}(\omega)} d V q_{X, O}\left(\rho, \theta_{p o l}, \omega, \vec{n}\right)}{\int d \Omega_{\vec{n}} \int_{S_{\mathrm{w}}}(d \vec{S} \cdot \vec{n})\left(1-R_{w}\right)+\int d \Omega_{\vec{n}} \int_{V_{x, O}^{\text {sec }}(\omega)} d V \kappa_{X, O}\left(\rho, \theta_{p o l}, \omega, \vec{n}\right)},
\end{gathered}
$$


$X$ and $O$ denote, respectively, extraordinary and ordinary waves; $q_{X, O}\left(\rho, \theta_{p o l}, \omega, \vec{n}\right)$ is power density of the ECR source; $\kappa_{\mathrm{X}, \mathrm{O}}\left(\rho, \theta_{\text {pol }}, \omega, \overrightarrow{\mathrm{n}}\right)$, the ECR absorption coefficient; $\rho$, effective radial coordinate (magnetic surface label); $\theta_{\text {pol }}$, poloidal angle; $\vec{k}$, wave vector; $\vec{n}=\vec{k} / k$, wave direction; $S_{w}$, area of vacuum chamber inner surface; $\mathrm{R}_{\mathrm{w}}$, reflection coefficient of the $\mathrm{EC}$ radiation from the wall. The integral $\int_{n} d \Omega_{n} \int_{S_{\mathrm{w}}}(d \vec{S} \cdot \overrightarrow{\mathrm{n}})\left(1-R_{\mathrm{w}}\right)$ is taken over the semi-sphere $(d \overrightarrow{\mathrm{S}} \cdot \overrightarrow{\mathrm{n}}) \geq 0 ; V_{X, O}^{\text {esc }}(\omega)$ is a part of the plasma volume where the ECR from superthermal electrons can propagate almost without absorption, with taking into account the wave cut-off.

The power density of ECR source can be calculated in the vacuum limit, but with allowance for the wave cut-off:

$$
\mathrm{q}_{\mathrm{x}, \mathrm{O}}\left(\rho, \theta_{\mathrm{pol}}, \omega, \theta_{\mathrm{k}}\right)=\int \mathrm{d}^{3} \mathrm{p} \mathrm{F}_{\mathrm{e}}^{\mathrm{hot}}\left(\rho, \theta_{\mathrm{pol}}, \mathrm{p}_{\|}, \mathrm{p}_{\perp}\right) \eta_{\mathrm{X}, \mathrm{O}}\left(\rho, \theta_{\mathrm{pol}}, \mathrm{p}_{\|}, \mathrm{p}_{\perp}, \omega, \theta_{\mathrm{k}}\right)
$$

$F_{e}^{\text {hot }}\left(\rho, \theta_{\text {pol }}, p_{\|}, p_{\perp}\right)$ is the superthermal electron VDF, $\eta_{X, O}$ is the emissivity for a single electron, calculated using the Schott-Trubnikov formula.

\section{Algorithm of VDF reconstruction}

To restore the VDF of superthermal electrons we solve an optimization problem that assumes determination of the function $F\left(\rho, \theta_{\text {pol }}, p_{\|}, p_{\perp}\right)$, for which the spectrum, $T_{\text {rad }}^{\text {calc }}(\omega)$, calculated in the frame of the proposed model, is as close as possible to the experimental ECR spectrum, $T_{\text {rad }}^{\exp }(\omega)$. In this paper we do not consider mechanisms other than EC emission for the observed spectra $\mathrm{T}_{\text {rad }}^{\exp }(\omega)$.

The inverse problem is linear, so one can divide the phase space into sectors $\left(\mathrm{p}_{\|}, \mathrm{p}_{\perp}, \rho, \theta_{\mathrm{pol}}\right)_{\mathrm{N}}(\mathrm{N}$ is the sector's number) and search for the contributions to $\mathrm{T}_{\text {rad }}^{\text {calc }}(\omega)$ from each sector, $\tilde{\mathrm{T}}_{\text {rad }}\left(\omega, \mathrm{p}_{\| \mathrm{N}}, \mathrm{p}_{\perp \mathrm{N}}, \rho_{\mathrm{N}}, \theta_{\text {polN }}\right)$ :

$$
\mathrm{T}_{\mathrm{rad}}^{\mathrm{calc}}(\omega)=\sum_{\mathrm{N}} \tilde{\mathrm{T}}_{\mathrm{rad}}\left(\omega, \mathrm{p}_{\| \mathrm{N}}, \mathrm{p}_{\perp \mathrm{N}}, \rho_{\mathrm{N}}, \theta_{\mathrm{polN}}\right) \cdot \mathrm{x}_{\mathrm{N}},
$$

where $\mathrm{x}_{\mathrm{N}}$ is the number of superthermal electrons in the $\mathrm{N}^{\text {th }}$ sector. The optimization problem is formulated as follows:

$$
\begin{gathered}
\min f, \quad \mathrm{x}_{\mathrm{N}} \geq 0, \\
f=f\left(\mathrm{~T}_{\mathrm{rad}}^{\exp }(\omega), \mathrm{T}_{\mathrm{rad}}^{\text {calc }}(\omega)\right),
\end{gathered}
$$

where $f$ is the objective function defined as the deviation of the calculated spectrum, $\mathrm{T}_{\text {rad }}^{\text {calc }}(\omega)$, from the experimental one, $\mathrm{T}_{\text {rad }}^{\exp }(\omega)$. Here we choose $f$ equal to the square of the Euclidean norm (L-2 norm), and $\mathrm{T}_{\text {rad }}$ functions are considered as vectors in the discretized $\omega$-space:

$$
f\left(\mathrm{~T}_{\text {rad }}^{\text {exp }}(\omega), \mathrm{T}_{\text {rad }}^{\text {calc }}(\omega)\right)=\left\|\mathrm{T}_{\text {rad }}^{\text {exp }}(\omega)-\mathrm{T}_{\text {rad }}^{\text {calc }}(\omega)\right\|_{2}^{2} .
$$

The problem (6)-(7) is an ill-posed inverse problem. The solution of this problem assumes such a reconstruction of the superthermal electronVDF which is accurate enough in predicting the mean characteristics of the VDF.

One can simplify the problem (5)-(6) by considering only the region of the phase space, which corresponds to trajectories of individual electrons with allowance for two conservation laws - the adiabatic invariant for an electron in a non-uniform magnetic field and the conservation of electron total energy. We denote with the 0 index the values of the electrons momentum at the point of minimum magnetic field at the trajectory (e.g., for trapped particles this point is the center of the banana trajectory). This allows the reduction of the dimensionality of optimization problem: now we 
search for the VDF which is averaged over poloidal angle at a fixed magnetic surface (e.g., for trapped particles this means the averaging of the VDF over banana trajectory). In this approach the electron momentum at a selected poloidal angle is uniquely determined by two parameters projections of the electrons momentum at the point of minimum magnetic field. For the reduced inverse problem we divide into sectors the reduced phase-space $\left(\mathrm{p}_{\| 0}, \mathrm{p}_{\perp 0}, \rho\right)$, so that equations (1)(3) for $T_{\text {rad }}^{\text {calc }}(\omega)$ turn into:

$$
\begin{gathered}
\mathrm{x}_{\mathrm{N}}=\mathrm{F}_{\mathrm{e}}^{\text {hot }}\left(\rho_{\mathrm{N}}, \mathrm{p}_{\| 0 \mathrm{~N}}, \mathrm{p}_{\perp 0 \mathrm{~N}}\right), \\
\int \rho \mathrm{d} \rho \int \mathrm{d}^{3} \mathrm{p} \rightarrow \sum_{\mathrm{N}} \rho_{\mathrm{N}} \Delta \rho_{\mathrm{N}} 2 \pi \mathrm{p}_{\perp 0 \mathrm{~N}} \Delta \mathrm{p}_{\perp 0 \mathrm{~N}} \Delta \mathrm{p}_{\| \mid 0 \mathrm{~N}} .
\end{gathered}
$$

In the reduced phase-space we need to calculate the radiation from an electron at a fixed magnetic surface with given projections of the momentum at the point of minimum magnetic field. The phasespace reduction needs the averaging which is defined by the operator:

$$
\int \frac{\mathrm{d} \theta_{\mathrm{pol}}}{2 \pi} \rightarrow \frac{1}{\tau_{\mathrm{b}}} \int \frac{\mathrm{ds}}{\left|\mathrm{v}_{\mathrm{s}}\right|},
$$

where $\mathrm{s}$ is the coordinate along the trajectory, $\tau_{\mathrm{b}}$ is the period of motion along the trajectory, $\mathrm{v}_{\mathrm{s}}$ is projection of electron velocity onto trajectory.

The algorithm of the reconstruction of the superthermal electron VDF is as follows.

- Space $\left(\mathrm{p}_{\| 0}, \mathrm{p}_{\perp 0}\right)$ for every magnetic surface $\rho$ is divided into sectors.

- Using the two conservation laws one can calculate the values of the electrons momentum $\mathrm{p}_{\|}\left(\theta_{\mathrm{pol}}\right), \mathrm{p}_{\perp}\left(\theta_{\mathrm{pol}}\right)$ at each point along the trajectory.

- $\tilde{\mathrm{T}}_{\mathrm{rad}}\left(\omega, \mathrm{X}_{\mathrm{N}}\right)$ is calculated using equations (1)-(3), and (4), with allowance for equation (9) and the bounce-averaging operator (10).

- Solving the optimization problem (6)-(7) returns the values $x_{N}=F_{e}^{\text {hot }}\left(\rho_{N}, p_{\| 0 N}, p_{\perp 0 N}\right)$.

\section{Inverse problem solution}

Here we present the results of the reconstruction of the superthermal electronVDF for the case of one of the observed spectra in the discharge № 36057 in tokamak T-10 (see curve №3 in Figure 2 for radiation spectral temperature in [1]). Main parameters of tokamak T-10 are as follows: major radius, $\mathrm{R}_{0}=1.5 \mathrm{~m}$, minor radius, $\mathrm{a}=0.3 \mathrm{~m}$, elongation, $\mathrm{k}_{\text {elong }}=1$, magnetic field on the axis, at $\mathrm{R}=\mathrm{R}_{0}$, is $\mathrm{B}_{0}=2.48 \mathrm{~T}$. We take the value of the wall reflection coefficient $\mathrm{R}_{\mathrm{w}}=0.6$.

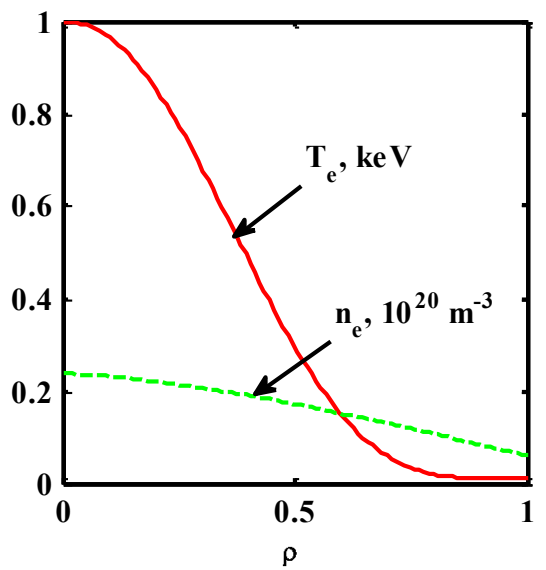

Fig. 1. Electron temperature and density profiles in the Ohmic discharge №36057 in tokamak T-10. 
The spectral region under interpretation includes the region lower than the $1^{\text {st }}(38-60 \mathrm{GHz})$ and the $2^{\text {nd }}$ harmonic cold resonance at the low magnetic field side $(95-120 \mathrm{GHz})$. The $1^{\text {st }}$ harmonic thermal radiation range $(60-90 \mathrm{GHz})$ and the $2^{\text {nd }}$ harmonic thermal radiation range $(>120 \mathrm{GHz})$ are excluded.

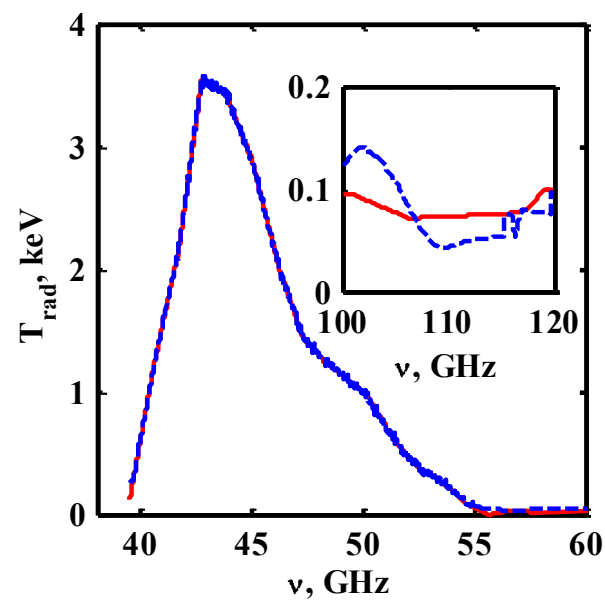

(a)

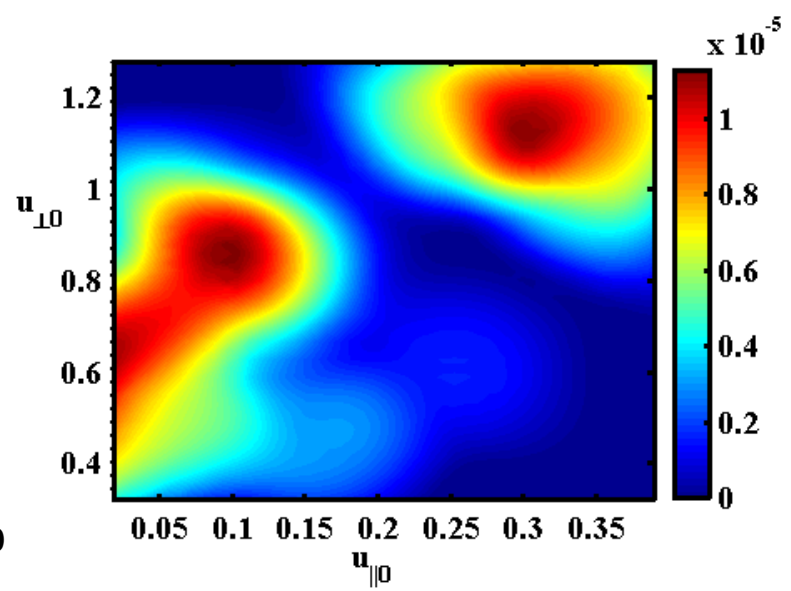

(b)

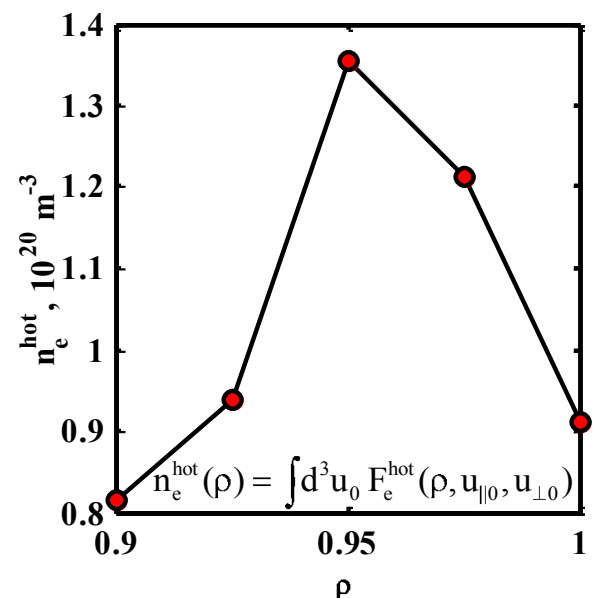

(c)

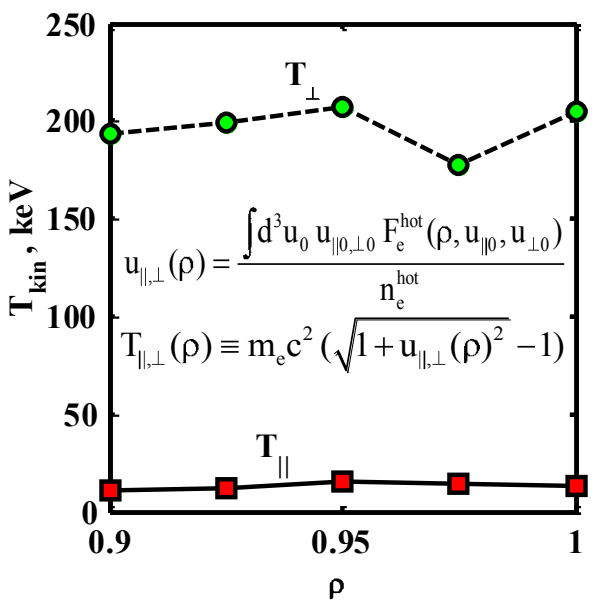

(d)

Fig. 2. (a) Radiation spectral temperatures: observed (red) (see curve №3 in Figure 2 in [1]) and obtained as a result of solving the optimization problem (6)-(7) (blue). Inset: similar spectra in the range between the highfrequency edge of the first harmonic of thermal ECR spectrum and the low-frequency edge of similar second harmonic spectrum. (b) The superthermal electron velocity distribution function, $x=F_{e}^{\text {hot }}\left(\rho, u_{\| 0}, u_{\perp 0}\right)\left(u=p / m_{e} c\right)$, for fixed magnetic surface $\rho=0.9$, reconstructed from the observed radiation temperature spectrum in Figure 2a. (c) The superthermal electron density profile, obtained by integration of velocity distribution function over momentum. (d) The profile of mean kinetic energy of superthermal electron, defined here for relativistic case (green circle markers - perpendicular to magnetic field component, red square markers - parallel component). Mean kinetic energies of superthermal electrons obtained by the additional spatial averaging are as follows: $<\mathrm{T}_{\text {kin }}>=213 \mathrm{keV},<\mathrm{T}_{\text {kinl }}>=13 \mathrm{keV},<\mathrm{T}_{\text {kin } \perp}>=196 \mathrm{keV}$. The ratio of superthermal electron density to the main plasma density within the layer is $\delta_{\text {hot }}=1.810^{-4}$. 


\section{Conclusions}

We formulated and solved an inverse problem for the reconstruction of the velocity distribution function (VDF) of superthermal electrons in parallel and perpendicular momenta, and in magnetic flux surfaces at the plasma edge. We consider the case when superthermal electrons are localized within $3 \mathrm{~cm}$ layer at the plasma edge as suggested by the experiments [1]. It is shown that in the Ohmic discharges in tokamak T-10 the superthermal electrons are the trapped particles at the low magnetic field side of toroid. Their mean kinetic energy lies in the range $\sim 150-200 \mathrm{keV}$, and the density fraction is about $\sim 10^{-4}$ relative to the main plasma at the edge.

The results obtained here for main characteristics of the superthermal electron VDF pose a question about possible mechanism of generating such superthermal electrons. Indeed, without ECRH the existence of high-energy strongly-trapped electrons seems to be unexpected. The observations in [2] of similar features of the low-frequency spectrum under similar conditions, without ECRH, was interpreted there as the EC radiation from superthermal electrons formed due to a maser effect. However, according to [2] these electrons were the circulating particles. Similar features of the low frequency spectrum have recently been observed in tokamak FT-2 [5]. Therefore, the question about possible mechanism of the formation of high-energy trapped electrons in the Ohmic discharges, which is beyond the scope of the present paper, seems to be of interest not only for tokamak T-10, but also for the experiments in other devices with low plasma density at the periphery.

The authors are grateful to V.I. Ilgisonis, A.V. Timofeev, V.L. Vdovin, V.A. Vershkov for fruitful discussions of the results. The work is supported by the State Corporation ROSATOM under the contract № H.4a.52.90.12.1006 and the Russian Federation Presidential Grants for State Support of Leading Scientific Schools (grant no. NSh_4361.2012.2).

\section{References}

1. V.I. Poznyak, et al., Problems of Atomic Science and Engineering, Ser. Thermonuclear Fusion (in Russian) 4, 90-110 (2011) (abstract in English)

2. B. Kurzan, K.H. Steuer, W. Suttrop, Rev. Sci. Instrum. 68, 423-426 (1997)

3. K. Kato, I.H. Hutchinson, Phys. of Fluids 30, 3809-3820 (1987)

4. S. Ide, K. Ogura, H. Tanaka, M. Iida, et al., Nucl. Fusion 29, 1325-1338 (1989)

5. S.I. Lashkul, et al., Zvenigorod XXXIX Intl conf. on plasma physics and controlled fusion (2012)

6. I.H. Hutchinson, K. Kato, Nucl. Fusion 26, 179-191 (1986)

7. I. Klimanov, PhD Thesis, (Lausanne, EPFL, 2005)

8. S. Tamor, Science Applications Report SAI-023-81-189LJ/LAPS-72 (1981)

9. A.B. Kukushkin, 14th IAEA Conference on Plasma Physics and Controlled Nuclear Fusion Research (1992)

10. K.V. Cherepanov, A.B. Kukushkin, 20th IAEA Fusion Energy Conference (2004)

11. A.B. Kukushkin, P.V. Minashin, 36th EPS Conference on Plasma Physics ( 2009)

12. A.B. Kukushkin, P.V. Minashin, A.R. Polevoi, Plasma Phys. Reports 38, 211-220 (2012) 